\title{
Ovarian Dermoid Cyst with Angiosarcoma
}

National Cancer Institute

\section{Source}

National Cancer Institute. Ovarian Dermoid Cyst with Angiosarcoma. NCI Thesaurus.

Code C40011.

A secondary angiosarcoma that has developed in an ovarian dermoid cyst. 